Canadian

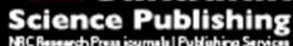

Applied Physiology, Nutrition, and Metabolism Physiologie appliquée, nutrition et métabolisme

\title{
A systematic upregulation of nuclear and mitochondrial genes is not present in the initial post-exercise recovery period in human skeletal muscle
}

\begin{tabular}{|r|l|}
\hline Journal: & Applied Physiology, Nutrition, and Metabolism \\
\hline Manuscript ID & apnm-2016-0455.R2 \\
\hline Manuscript Type: & Article \\
\hline Date Submitted by the Author: & 08-Dec-2016 \\
\hline Complete List of Authors: & $\begin{array}{l}\text { Scribbans, Trisha; University of Manitoba, Faculty of Kinesiology and } \\
\text { Recreation Management } \\
\text { Edgett, Brittany; Queen's University, School of Kinesiology and Health } \\
\text { Studies } \\
\text { Bonafiglia, Jacob; Queen's University, School of Kinesiology and Health } \\
\text { Studies } \\
\text { Baechler, Brittany; University of Waterloo, Department of Kinesiology } \\
\text { Quadrilatero, Joe; University of Waterloo, } \\
\text { Gurd, Brendon J.; Queens University, }\end{array}$ \\
\hline Keyword: & $\begin{array}{l}\text { exercise physiology < exercise, exercise metabolism < exercise } \\
\text { metabolism, mitochondrial metabolism < metabolism, muscle physiology < } \\
\text { muscle, skeletal muscle metabolism < muscle }\end{array}$ \\
\hline &
\end{tabular}




\title{
A systematic upregulation of nuclear and mitochondrial genes is not present in the
} initial post-exercise recovery period in human skeletal muscle

Trisha D. Scribbans ${ }^{1 *}$, Brittany A. Edgett ${ }^{1}$, Jacob T. Bonafiglia ${ }^{1}$, Brittany L. Baechler ${ }^{2}$, Joe Quadrilatero $^{2}$ and Brendon J. Gurd ${ }^{1}$.

\author{
${ }^{1}$ School of Kinesiology and Health Studies, \\ Queen's University, Kingston, Ontario, Canada \\ ${ }^{2}$ Department of Kinesiology, University of Waterloo, \\ Waterloo, Ontario, Canada
}

Trisha D. Scribbans (trisha.scribbans@umanitoba.ca), Brittany A. Edgett (b.edgett@queensu.ca), Jacob T. Bonafiglia (0jtb1@queensu.ca), Brittany L. Baechler (brittany.baechler@uwaterloo.ca), Joe Quadrilatero (jquadril@uwaterloo.ca), Brendon J. Gurd (gurdb@queensu.ca)

Corresponding Author:

Trisha D. Scribbans, PhD, C.A.T.(C)

Telephone: 204-272-1699

Fax: 204-474-7634

Email: trisha.scribbans@umanitoba.ca

${ }^{*}$ Current affiliation for T. Scribbans: Faculty of Kinesiology and Recreation Management, University of Manitoba, Winnipeg, Manitoba, R3T 2N2, Canada 


\section{Abstract}

The purpose of the current investigation was to determine if an exercise-mediated upregulation of nuclear and mitochondrial-encoded genes targeted by the transcriptional coactivator peroxisome-proliferator-activated receptor gamma co-activator-1 alpha (PGC-1 $\alpha)$ occurs in a systematic manner following different exercise intensities in humans. Ten recreationally active males (Age: $23 \pm 3$ yrs; $\mathrm{VO}_{2}$ peak: $41.8 \pm 6.6 \mathrm{~mL} / \mathrm{kg} / \mathrm{min}$ ) completed two acute bouts of work-matched interval exercise at $\sim 73 \%$ (LO) and $\sim 100 \%$ (HI) of work rate at $\mathrm{VO}_{2}$ peak in a randomized cross-over design. Muscle biopsies were taken before (Pre), immediately after (Post), and 3 hours into recovery (3hr) following each exercise bout. A main effect of time $(p<0.05)$ was observed for glycogen depletion. PGC-1 $\alpha$ mRNA increased following both conditions and was significantly $(p<0.05)$ higher following HI compared to LO (PGC-1 $\alpha$, LO: $+442 \%$ vs. HI: $+845 \%$;). PDK4 mRNA increased following LO whereas PPAR $\alpha$, NRF1, and CS increased following HI. However, a systematic upregulation of nuclear and mitochondrial-encoded genes was not present as TFAM, COXIV, COXI, COXII, ND1 and ND4 mRNA were unchanged. However, changes in COXI, COXII, ND1and ND4 mRNA were positively correlated following LO and COXI, ND1, and ND4 were positively correlated following HI, which suggests mitochondrial-encoded gene expression was coordinated. PGC-1 $\alpha$ and ND4 mRNA, as well as PGC-1 $\alpha$ mRNA and the change in muscle glycogen, were positively correlated in response to LO. The lack of observed systematic upregulation of nuclear- and mitochondrial-encoded genes suggests that exercise-induced upregulation of PGC-1 $\alpha$ targets are differentially regulated during the initial hours following acute exercise in humans.

Key words: exercise physiology, exercise metabolism, mitochondrial metabolism, muscle physiology, skeletal muscle metabolism 


\section{Introduction}

Peroxisome-proliferator-activated receptor gamma co-activator-1 alpha (PGC-1 $\alpha$ ) is a transcription co-factor that is frequently designated as the 'master regulator of mitochondrial biogenesis' (Puigserver et al. 1998, Safdar et al. 2011, Scarpulla 2011). As a transcriptional coactivator, PGC-1 $\alpha$ interacts with and co-activates numerous transcription factors and nuclear receptors that subsequently bind to the promoters of both PGC-1 $\alpha$ itself (Handschin et al. 2003) and nuclear-encoded mitochondrial genes to increase their expression (Scarpulla 2011). Specifically, PGC-1 $\alpha$ co-activates nuclear respiratory factor-1 (NRF1), NRF2 (Wu et al. 1999), myocyte enhancer factor 2 (MEF2) (Handschin et al. 2003), estrogen-related receptors (ERR's) and thyroid receptor (Puigserver et al. 1998). Importantly, NRF1 and NRF2 activate the mitochondrial transcription factor A (TFAM) promoter, a nuclear-encoded factor that translocates to the mitochondria and initiates the transcription and replication of mitochondrial DNA (mtDNA) (Virbasius and Scarpulla 1994, Wu et al. 1999, Ramachandran et al. 2008). Therefore, PGC-1 $\alpha$ is believed to play a central, albeit non-obligatory (Leick et al. 2007, Rowe et al. 2012), role in coordinating the regulation of nuclear and mitochondrial-encoded gene expression necessary for mitochondrial biogenesis.

In response to acute exercise, the transcriptional activity of PGC-1 $\alpha$ is believed to be increased in part as a result of changes in its sub-cellular localization (Wright et al. 2007, Little et al. 2010, 2011, Safdar et al. 2011). Specifically, PGC-1 $\alpha$ translocates to the nucleus where it increases the expression of nuclear-encoded mitochondrial genes following acute contractile activity in mice (Wright et al. 2007, Safdar et al. 2011) and following endurance and highintensity interval training in humans (Little et al. 2010, 2011). In addition, acute endurance exercise induces the translocation of PGC-1 $\alpha$ to the mitochondrial matrix in mice (Safdar et al. 
2011) and humans (Smith et al. 2013) where it complexes with TFAM to increase mitochondrialencoded gene expression (Safdar et al. 2011). Collectively, these findings suggest that PGC-1 $\alpha$ coordinates the integrated induction of mitochondrial biogenesis in response to acute exercise via its redistribution to the nucleus and mitochondria; a hypothesis that is supported by the systematic upregulation of mitochondrial- and nuclear-encoded genes following acute exercise in mice (Safdar et al. 2011). Surprisingly, while the expression of both nuclear- and mitochondrialencoded genes are acutely up-regulated following supramaximal interval exercise in humans (Psilander et al. 2010, Little et al. 2011), genes targeted by PGC-1 $\alpha$ are not systematically increased following lower intensities of exercise (Barrès et al. 2012, Stepto et al. 2012, Popov et al. 2015). These results raise the possibility that the coordinated induction of nuclear and mitochondrial gene expression may only occur in human skeletal muscle following intensities of exercise where activation of PGC-1 $\alpha$ is high. Given that the expression of PGC-1 $\alpha$ increases in an intensity-dependent manner up to $\mathrm{VO}_{2}$ peak (Egan et al. 2010, Nordsborg et al. 2010, Edgett et al. 2013), it is reasonable to hypothesize that if a coordinated expression of nuclear- and mitochondrial-encoded genes targeted by PGC-1 $\alpha$ occurs in human skeletal muscle, it likely occurs following near-maximal intensity exercise.

Therefore, the purposes of the current investigation were to determine: 1) if the exercisemediated upregulation of nuclear- (PGC-1 $\alpha$, PPAR $\alpha$, NRF1, TFAM, PDK4, CS, COXIV) and mitochondrial- (COXI, COX II, ND1, ND4) encoded genes targeted by PGC-1 $\alpha$ occurs in a systematic manner, and 2) if higher intensities of exercise are required to induce a systematic upregulation of nuclear and mitochondrial genes.

\section{Materials and Methods}

Experimental design 
In order to investigate the effect of submaximal and maximal intensity exercise on the expression of nuclear- and mitochondrial-encoded genes, participants completed two acute bouts of work-matched interval exercise at $\sim 73 \%$ (low; LO) and $\sim 100 \%$ (high; HI) of work rate (WR) at $\mathrm{VO}_{2}$ peak in a randomized cross-over design. All experimental procedures were approved by the Health Sciences Human Research Ethics Board at Queen's University and conformed to the Declaration of Helsinki. Verbal and written explanation of the experimental protocol and associated risks were provided to all participants prior to obtaining written informed consent.

\section{Participants}

Ten healthy males volunteered to participate in the study (participant characteristics are presented in Table 1). At the time of enrolment, all participants were recreationally active, but not involved in more than 3 hours of aerobic exercise (running, jogging, etc.) per week.

\section{Physiological testing}

During their first visit to the lab, participants completed a $\mathrm{VO}_{2}$ peak incremental ramp test to exhaustion on a cycle ergometer (Monark, Ergomedic 874E, Varberg, Sweden) and anthropometric measurements (height and weight) were obtained. Briefly, the ramp protocol consisted of five minutes of load-less cycling followed by a step increase to 80 watts (W) for one minute and subsequent increases in $\mathrm{WR}$ of $25 \mathrm{~W} \cdot \mathrm{min}^{-1}$ until volitional fatigue (determined by the inability of the participant to maintain a cadence of 60 RPM). Gas exchange was measured throughout the test with a metabolic cart (Moxus AEI Technologies, Pittsburgh, PA). RPM was collected continuously throughout the test. Absolute and relative $\mathrm{VO}_{2}$ peak, heart rate (HR) peak and WR peak were selected as the highest value of the same continuous 30 second average at the end of the test.

Experimental visits 
A minimum of 72 hours following the $\mathrm{VO}_{2}$ peak test, participants reported to the lab 30 minutes after consuming a standardized breakfast [plain bagel (190 kcal; $1 \mathrm{~g}$ fat, $36 \mathrm{~g}$ carbohydrate, $7 \mathrm{~g}$ protein) with $15 \mathrm{~g}$ of peanut butter (90 kcal; $8 \mathrm{~g}$ fat, $4 \mathrm{~g}$ carbohydrate, $3 \mathrm{~g}$ protein) and $200 \mathrm{~mL}$ of apple juice (90 kcal; $0 \mathrm{~g}$ fat, $22 \mathrm{~g}$ carbohydrate, $0 \mathrm{~g}$ protein)] at home. Participants were also given a standardized dinner to consume the night before [Stouffer's Sauté Sensations Country Beef Pot Roast (540 kcal; 56 g carbohydrate (CHO), 20 g fat, 14 g protein) and $500 \mathrm{~mL}$ of $2 \%$ milk ( $260 \mathrm{kcal} ; 12 \mathrm{~g} \mathrm{CHO}, 5 \mathrm{~g}$ fat, $9 \mathrm{~g}$ protein)], which was followed by an overnight fast $(\geq 12 \mathrm{~h}$ ) before consuming breakfast. Confirmation of completion of breakfast and dinner was sent to the investigators via text message. After arrival at the lab, a resting muscle biopsy (Pre) was taken from the vastus lateralis under superficial local anaesthesia (2\% lidocaine, with epinephrine) using the Bergstrom needle biopsy technique (Bergstrom 1975) adapted with suction. An additional incision was made in the same leg, and covered with sterile gauze, approximately $2 \mathrm{~cm}$ distal to the resting biopsy site to allow for immediate removal of the post-exercise muscle sample. Participants then rested in the lab for 44 or 38 minutes before completing a bout of high-intensity interval training (HIT) at either $\sim 100$ or $73 \%$ of WR at $\mathrm{VO}_{2}$ peak, respectively. Immediately following exercise, a second muscle biopsy was taken (Post). A final biopsy was taken 3 hours post-exercise (3hr) from the same leg following analgesia and an additional incision $\sim 2 \mathrm{~cm}$ distal to the second biopsy site. Experimental visits were separated by a minimum of 7 days (maximum 17 days) and were performed on the same cycle ergometer. One portion of each muscle biopsy was embedded in O.C.T (Tissue-Tek, Sakura Finetek, CA, USA) and frozen in liquid nitrogen-cooled isopentane for determination of glycogen content. The remaining muscle sample was frozen immediately in liquid nitrogen and stored at $-80^{\circ} \mathrm{C}$ until analyzed for changes in gene expression via real-time PCR. 


\section{Exercise protocols}

Both HIT protocols consisted of one minute intervals separated by one minute of loadless cycling at a cadence of the participant's choosing on a cycle ergometer. The low-intensity interval protocol (LO) consisted of 11 intervals at 80 RPM against a load corresponding to $\sim 73 \%$ of peak aerobic WR. The high-intensity interval protocol (HI) consisted of 8 intervals at 80 RPM against a load corresponding to $\sim 100 \%$ of peak aerobic WR. External work and heart rate (HR) were recorded at the beginning and end of each interval (Polar Team ${ }^{2}$ Pro, La-chine, Quebec, Canada). Participants were asked to maintain a cadence of 80 RPM during each interval; however, if RPM fell below or above 80, intervals were added or removed such that the target amount of work achieved in both HIT sessions were the same. Actual and prescribed kilocalories and the number of intervals performed is presented in Figure $1 \mathrm{~A}$ and $\mathrm{B}$, respectively. HR data is presented in Figure $1 \mathrm{C}$.

\section{Determination of muscle glycogen content}

Glycogen content was determined using the Periodic Acid Schiff (PAS) (Quadrilatero et al. 2010). Images were acquired with a bright field Nikon microscope linked to a PixelLink digital camera. Individual images were taken across the entire muscle cross-section and assembled into a composite panoramic image using Microsoft Image Composite Editor (ICE) (Microsoft, Redmond, WA, USA). Image analysis was performed in ImageJ (National Institute of Health, Bethesda, Maryland, USA) by converting color images to 8-bit, and calculated by subtracting background staining from individual fibres. Approximately 100 fibres were randomly selected from each compiled image and the mean optical density (OD) of the fibers was used as an indication of muscle glycogen content.

\section{Determination of gene expression}


RNA extraction and real-time PCR were performed on Pre and 3hr samples for both LO and HI conditions. These two time-points were chosen as both previous investigations reporting a coordinated increase in the expression of nuclear- and mitochondrial-encoded genes in mice measured changes in gene expression prior to, and 3 hours following acute exercise (Safdar et al. 2011, Saleem and Hood 2013). Further, in humans, increases in PGC-1 $\alpha$ gene expression peak between 2 and 5 hours post exercise (Pilegaard et al. 2003, Nordsborg et al. 2010) and increases in both nuclear and mitochondrial encoded genes are present 3 hours post SIT (Little et al. 2010). RNA was extracted using a modified version of the single-step method by guanidinium thiocyanate-phenol-chloroform extraction (Chomczynski and Sacchi 2006). The purified RNA pellet was dissolved in RNase and DNase-free ultrapure water then quantified spectrophotometrically at $260 \mathrm{~nm}$ using a Take3 Plate (Biotek, Winooski, VT, USA). Protein contamination was assessed by measuring absorbance at $280 \mathrm{~nm}$. Samples had an average 260:280 ratio of $1.97 \pm 0.02$ (mean $\pm \mathrm{SD}$ ). One microgram of resulting RNA was reverse transcribed using the QuantiTect Reverse Transcription Kit (Qiagen, Mississauga, Canada). Transcript levels were determined on an ABI 7500 Real Time PCR System (Foster City, CA, USA) using the following protocol: 1 cycle at $95^{\circ} \mathrm{C}$ for 15 minutes, 40 cycles of $95^{\circ} \mathrm{C}$ for 15 seconds, 30 seconds at $59^{\circ} \mathrm{C}$, and $72^{\circ} \mathrm{C}$ for 36 seconds, followed by a dissociation curve to assess specificity of the reaction. Primer set efficiencies were determined using real-time PCR with an appropriate cDNA dilution series prior to sample analysis. Average primer set-specific efficiencies (Livak and Schmittgen 2001) were $\mathrm{E}=1.99 \pm 0.06$ (mean $\pm \mathrm{SD}$ ). All samples were run in duplicate $25 \mu \mathrm{l}$ reactions containing: 1-50 ng cDNA (gene dependant), $0.58 \mu \mathrm{M}$ primers, and GoTaq PCR Master Mix (Promega, Madison, WI). No-template controls were run with water in place of cDNA to ensure the absence of contamination. Primer sequences are provided 
in Table 2. Importantly, the PGC-1 $\alpha$ primer set used in the current study targets only the full length PGC-1 $\alpha$ isoforms (PGC-1 $\alpha 1$ [PGC-1 $\alpha-a$; canonical], PGC- $1 \alpha-b$ and PGC-1 $\alpha-c)$. All RNA data are expressed relative to TATA-binding protein (TBP), which was stable across all states with no difference in the raw $\mathrm{C}_{\mathrm{T}}$ values observed between conditions or Pre and 3 hours postexercise (LO, Pre: $27.59 \pm 0.36,3 \mathrm{hr}: 27.36 \pm 0.12$; HI, Pre: $27.36 \pm 0.24,3 \mathrm{hr}: 27.43 \pm 0.25$ ).

\section{Statistical analysis}

A two-way, repeated measures analysis of variance (ANOVA) was used to compare the effect of condition (LO vs. HI) and time (Pre vs. Post) on glycogen depletion, and the effect of condition (LO vs. HI) and work (actual vs. prescribed). Differences in HR between the HI and LO exercise bouts were determined using paired $t$-tests for intervals 1-8. Paired $t$-tests were performed on linear data using the $\Delta \mathrm{Ct}$ and $\Delta \Delta \mathrm{Ct}$ methods (Livak and Schmittgen 2001) to compare the effect of exercise on mRNA expression in both LO and HI conditions, and also to compare differences in mRNA expression between both exercise intensities, respectively. Given that the genetic diversity of humans and the large degree of heterogeneity in responses to exercise between individuals (Bouchard and Rankinen 2001, Vollaard et al. 2009) could potentially mask a coordinated response at the group level, we also examined the relationship between individual changes in nuclear- and mitochondrial-encoded genes. Bivariate correlations were performed to determine if changes in PGC-1 $\alpha$, pyruvate dehydrogenase kinase isozyme 4 (PDK4), peroxisome proliferator activated receptor alpha (PPAR $\alpha), \mathrm{NRF} 1$, citrate synthase (CS), cytochrome $c$ oxidase (COX) subunit I (COXI), II, (COXII), and COXIV, and NADH dehydrogenase subunit 1 (ND1) and 4 (ND4) gene expression and the change in glycogen content from pre- to post-exercise were related in response to LO or HI (Table 3). Pearson correlation coefficient $(r)$ effect sizes were classified as small $(r= \pm 0.1)$, medium $(r= \pm 0.3)$, or 
large $(r= \pm 0.5)$ (Field 2013). All statistical analyses were performed using the statistical program SPSS 20.0 (SPSS Inc., Chicago, IL, USA). All figures were made using GraphPad Prism v 5.01 (GraphPad Software Inc., La Jolla, CA). Statistical significance was accepted at $p<$ 0.05 .

\section{Results}

\section{Exercise bout characteristics and glycogen depletion}

External work performed was not different between groups (Figure 1A) and, as designed, participants completed significantly $(p<0.05)$ more intervals during the LO than HI condition (Figure 1B). HR at the end of intervals 2-8 was significantly higher during HI than LO (Figure 1C). A main effect of time $(p<0.05)$ was observed for glycogen depletion, with no difference between conditions (Figure 2A). Representative glycogen images are presented in Figure 2B. Nuclear and mitochondrial encoded gene expression

PGC-1 $\alpha$ mRNA increased following both acute exercise bouts and was significantly $(p<$ 0.05) higher following HI (LO: $+442 \%$ vs. HI: $+845 \%$; Figure $3 \mathrm{~A}$ ). PDK4 mRNA increased significantly $(p<0.05)$ following LO, whereas PPAR $\alpha$, NRF1, and CS mRNA increased following HI. However, changes in PPAR $\alpha$, NRF1, and CS mRNA expression was not different between exercise bouts (PPAR $\alpha$ : LO: $+20 \%$ vs. HI: $+22 \%$; NRF1: LO: $+8 \%$ vs. HI: $+20 \%$; CS: LO: $+8 \%$ vs. HI: $+12 \%$; Figure $3 \mathrm{~A}$ ). The expression of the nuclear-encoded genes, TFAM, CS, and COXIV were unchanged following both acute exercise bouts as were the mitochondrialencoded genes, COXI, COXII, ND1 and ND4 (Figure 3A).

Coordinated expression of nuclear and mitochondrial-encoded genes

Following LO, COXIV positively correlated with PPAR $\alpha(r=0.83, p=0.00)$ and NRF1 $(r=0.66, p=0.04)$. Following HI, PPAR $\alpha$ correlated with CS $(r=0.85, p=0.00)$, and NRF1 
positively correlated with PDK4 $(r=0.80, p=0.01)$ and CS $(r=0.69, p=0.03)$. No other significant correlations were observed between the changes in any of the nuclear encoded genes examined. Positive correlations were observed between COXIV, COXI, COXII, ND1 and ND4 mRNA expression in response to LO and HI (Table 3). Mitochondrial-encoded gene expression for individual participants in response to $\mathrm{LO}$ and $\mathrm{HI}$ are presented in Figure $3 \mathrm{~B}$ and $\mathrm{C}$, respectively. In response to LO only, positive correlations were present between PGC-1 $\alpha$ and ND4 $(r=0.64, p=0.05), \operatorname{PPAR} \alpha$ and COXI $(r=0.74, p=0.01)$, and NRF1 and COXI $(r=0.70$, $p=0.03) \mathrm{ND} 1(r=0.74, p=0.02)$, and ND4 $(r=0.69, p=0.03)$. Additionally, while PGC-1 $\alpha$ expression was positively correlated with the change in muscle glycogen content observed in response to LO $(r=0.73, p=0.02)$, but not HI $(r=-0.18, p=0.62)$. No other significant relationships involving mitochondrial-encoded gene expression were observed ${ }^{1}$. All significant correlations had large effect sizes.

\section{Discussion}

In the initial hours following acute exercise a systematic upregulation of nuclear and mitochondrial-encoded genes is observed in mouse skeletal muscle (Safdar et al. 2011, Saleem and Hood 2013). The present study examined whether a similar systematic induction of nuclearand mitochondrial-encoded genes targeted by PGC- $1 \alpha$ is present in the early post-exercise response period in human skeletal muscle. Additionally, we examined whether the magnitude of change in nuclear and mitochondrial gene expression was exercise intensity-dependent. Unlike the response present in mice following exercise (Safdar et al. 2011, Saleem and Hood 2013), we failed to observe a systematic upregulation of nuclear and mitochondrial encoded genes following either LO or HI exercise. Interestingly, while we did not observe an increase in the

\footnotetext{
1 See Supplemental Tables S1 and S2 for complete correlation matrices.
} 
expression of many of the genes examined 3 hours following exercise (TFAM, COXIV, COXI, ND1, and ND4), analysis of individual participant responses suggests that coordinated regulation may be present amongst the mitochondrial-encoded genes examined (COXI, COXII, ND1 and ND4).

Nuclear-and mitochondrial-encoded gene expression are not systematically upregulated in human skeletal muscle in the early post-exercise period

In response to acute exercise, PGC-1 $\alpha$ translocates to the nucleus and mitochondrial matrix in mice (Safdar et al. 2011, Saleem and Hood 2013) and human skeletal muscle (Little et al. 2010, 2011, Smith et al. 2013) where it is proposed to coordinate mitochondrial biogenesis by controlling the expression of nuclear- and mitochondrial-encoded genes (Puigserver et al. 1998, Wu et al. 1999, Handschin et al. 2003, Mootha et al. 2004, Ramachandran et al. 2008, Safdar et al. 2011). Acutely exercising mice results in a concomitant increase in nuclear and mitochondrial PGC-1 $\alpha$ content, and an upregulation of nuclear, (PGC-1 $\alpha$, PDK4, NRF1, TFAM, CS, COXIV, cytochrome c) and mitochondrial-encoded gene expression (COXI, ND1, ND4) immediately after exercise, and 3 hours into recovery (Safdar et al. 2011, Saleem and Hood 2013). Given that the expression of PGC-1 $\alpha$ is greatest at near-maximum intensities (Edgett et al. 2013), and PGC$1 \alpha$ target genes are upregulated in the initial hours following supramaximal (Psilander et al. 2010, Little et al. 2011), but not moderate exercise intensities (Barrès et al. 2012, Stepto et al. 2012, Popov et al. 2015), we hypothesized that an upregulation of nuclear- and mitochondrialencoded genes would be present in response to an acute bout of near-maximal intensity exercise (HI; 100\% $\mathrm{VO}_{2}$ peak WR). Contrary to our hypothesis, and despite the greater increase in PGC$1 \alpha$ expression (i.e. $\mathrm{HI}>\mathrm{LO}$ ), we did not observe a systematic upregulation of nuclear and mitochondrial genes targeted by PGC-1 $\alpha$ following either intensity of exercise. 
While increases in the expression of nuclear and mitochondrial genes have been reported in human skeletal muscle following repeated $30 \mathrm{sec}$ 'all out' bursts (sprint interval training; SIT) (Psilander et al. 2010, Little et al. 2011), moderate intensity exercise does not appear to have this effect (Cartoni et al. 2005, Nordsborg et al. 2010, Psilander et al. 2010, Barrès et al. 2012, Stepto et al. 2012, Bartlett et al. 2013, Jensen et al. 2015, Popov et al. 2015). Interestingly, investigations in mice reporting a systematic upregulation of nuclear and mitochondrial genes immediately after, and 3 hours following exercise employed large doses of endurance exercise (90 minutes of treadmill running at $15 \mathrm{~m} / \mathrm{min}$ ) (Safdar et al. 2011, Saleem and Hood 2013). Further, the coordinated expression of nuclear and mitochondrial genes previously reported in mice (Safdar et al. 2011) and humans (Little et al. 2011) were accompanied by increases in nuclear PGC-1 $\alpha$ protein content. Therefore, it is plausible that of our lack of observed systematic upregulation of nuclear and mitochondrial genes may be the result of PGC-1 $\alpha$ not being redistributed to the nucleus. Future investigations should attempt to determine changes in the cellular redistribution of PGC-1 $\alpha$ following acute exercise of differing intensities and doses and their impact on the expression of nuclear- and mitochondrial-DNA encoded genes.

It is also possible that the recreational activity status of our participants may have contributed to the lack of coordinated induction of PGC-1 $\alpha$ targets that has been previously observed in response to supramaximal interval exercise in elite cyclists (Psilander et al. 2010). However, while training status is an important factor to consider in future studies, especially in light of recent demonstrations that the genetic response to exercise can be altered following training (Serpiello et al. 2012, Stepto et al. 2012), the bulk of the literature (Nordsborg et al. 2010, Stepto et al. 2012, Popov et al. 2015) and the results of the current study suggests that the early induction of genes targeted by PGC-1 $\alpha$ is not coordinated. These findings, together with 
the observations of the current investigation, suggest that the early response of individual genes targeted by PGC-1 $\alpha$ may be regulated differently during, and following supramaximal exercise intensities or larger exercise doses such that a coordinated response may exist following these conditions.

Alternatively, it is possible that the time-point measured in the current study failed to capture a systematic upregulation of nuclear- and mitochondrial-encoded genes that occurred later in the post-exercise period. Supporting this contention, the expression of nuclear-encoded genes targeted by PGC-1 $\alpha$ demonstrate divergent temporal patterns of upregulation following exercise in humans with several genes (including PGC-1 $\alpha$ ) being rapidly upregulated, and others failing to increase until 24 hours post-exercise (Cartoni et al. 2005, Perry et al. 2010). While this suggests that coordinated increases of PGC-1 $\alpha$ target genes may occur over time following exercise, our results, and those of others (Perry et al. 2010, Barrès et al. 2012, Stepto et al. 2012, Percival et al. 2015, Popov et al. 2015) suggest that regulation of mitochondrial gene expression is considerably more complicated than currently understood. Specifically, it would appear that PGC-1 $\alpha$ is controlled by mechanisms that result in its rapid induction following exercise (Norrbom 2003, Little et al. 2010, 2011, Perry et al. 2010, Edgett et al. 2013, Jensen et al. 2015), while other PGC-1 $\alpha$ targets (both nuclear and mitochondrial encoded) are upregulated by different mechanisms that follow a longer time course.

The demonstration that PGC-1 $\alpha$ is not required for exercise induced mitochondrial biogenesis (Leick et al. 2007, Rowe et al. 2012) raises the possibility that the transcriptional response to exercise in muscle is under the control of a host of transcription factor co-activators and transcription factors that combine to determine the transcriptional response to exercise. Further, while the mitochondrial genes examined in the current investigation were not 
systematically upregulated in response to exercise it is possible that genes outside of the PGC-1 $\alpha$ targets measured here may have responded in a systematic manner. In order to fully characterize the transcriptional response of the entire mitochondrial genome to acute exercise future research should utilize high-throughput techniques such as microarrays.

In summary, the coordinated, systematic, upregulation of nuclear- and mitochondrialencoded genes that occurs in mouse skeletal muscle following exercise (Safdar et al. 2011, Saleem and Hood 2013) appears not to be conserved in humans. The lack of a systematic upregulation of PGC-1 $\alpha$ target genes in the current study highlights the need for a reappraisal of the current understanding of the coordination of mitochondrial biogenesis via PGC-1 $\alpha$, and the need for more research examining mechanisms controlling both PGC-1 $\alpha$ gene expression and the expression of other PGC-1 $\alpha$ target genes.

The impact of exercise intensity and intramuscular glycogen content on PGC-1 $\alpha$ expression

Comparable reductions in muscle glycogen content were present in response to an acute bout of $\mathrm{HI}$ and LO exercise, which suggests that the overall metabolic stress imposed on the skeletal muscle by the two different exercise bouts was similar. Despite this observation, PGC$1 \alpha$ expression was greater following the HI intensity exercise, and is consistent with previous reports (Egan et al. 2010, Edgett et al. 2013). This suggests that factors regulating PGC-1 $\alpha$ expression/activity outside of those resulting from differences in the magnitude of metabolic stress imposed on the exercising muscle by different exercise intensities, influences PGC-1 $\alpha$ expression (e.g. hormonal response, sub-cellular distribution, etc. (Benton et al. 2008)).

Interestingly, in response to LO, PGC-1 $\alpha$ expression was correlated with reductions in glycogen content, which suggests that individual changes in glycogen content may influence PGC-1 $\alpha$ activity at this intensity. This is supported by reports that the amount of, or localization of 
glycogen particles can directly or indirectly impact intracellular signaling and gene expression (Philp et al. 2012, 2013). Collectively, these findings suggest that PGC-1 $\alpha$ expression increases with exercise intensity, and that this response may be mediated in part by changes in glycogen content in response to sub-maximal intensity exercise.

Individual coordinated response in the expression of select mitochondrial genes

Interestingly, while a coordinated upregulation of nuclear and mitochondrial PGC-1 $\alpha$ target genes was not observed in the current study, correlational analysis revealed a coordinated expression of only the mitochondrial encoded genes following both LO (COXI, COXII, ND1 and ND4) and HI (COXI, COXII, ND1 and ND4). Thus, while evidence of a coordinated increase in mitochondrial genes was not present at a group level, and fold changes in gene expression were at best modest at an individual level (all $<1.8$ fold), the effect sizes of the correlations between mitochondrial genes were all in excess of $r=0.6$. While the biological significance of these associations are not currently known, these findings, coupled with the lack of association of PGC-1 $\alpha$ expression with these genes suggests that the expression of mitochondrial encoded genes are under the control of a common mechanism that is different from the mechanism controlling PGC-1 $\alpha$ expression. Translocation of PGC-1 $\alpha$ to the mitochondrial matrix is a likely candidate given observations that PGC- $1 \alpha$ redistributes to the mitochondrial matrix in response to exercise in humans (Smith et al. 2013). The tumour suppressor protein p53 is another potential candidate as it has been demonstrated to translocate to the mitochondrial matrix with a concomitant increase in mitochondrial encoded gene expression in response to acute exercise in mice (Saleem and Hood 2013); however, a recent investigation in humans reported no change in mitochondrial p53 content following 60 minutes of cycling at $\sim 70 \% \mathrm{VO}_{2}$ peak (Tachtsis et al. 2016). While the mechanism(s) responsible for the apparent 
coordination of mitochondrial-encoded gene expression following exercise in humans is unknown, identification of these mechanisms and their interaction with PGC-1 $\alpha$ and/or other transcription factors are an important area for future investigation.

\section{Conclusions and future directions}

Dissimilar to mice, a systematic upregulation of nuclear- and mitochondrial-encoded genes is not present in human skeletal muscle in the initial hours following an acute bout of either LO or HI exercise. The disassociation between PGC-1 $\alpha$ expression and the expression of other PGC-1 $\alpha$ targeted mitochondrial genes suggests that exercise induced upregulation of gene expression is differentially regulated during the initial hours following acute exercise in humans. However, the coordinated expression of mitochondrial-encoded genes suggests that an unknown regulatory mechanism coordinates their induction, and may link the expression of nuclear- and mitochondrial-encoded genes in response to acute exercise in humans. Future investigations should attempt to describe the unique mechanism(s) regulating the expression of mitochondrial genes and the time-course of their expression in human skeletal muscle in response to exercise.

\section{Disclosure}

The authors have no conflict of interest to disclose. 


\section{References}

Barrès, R., Yan, J., Egan, B., Treebak, J.T., Rasmussen, M., Fritz, T., Caidahl, K., Krook, A., O’Gorman, D.J., and Zierath, J.R. 2012. Acute Exercise Remodels Promoter Methylation in Human Skeletal Muscle. Cell Metab. 15(3): 405-411. doi:10.1016/j.cmet.2012.01.001.

Bartlett, J.D., Louhelainen, J., Iqbal, Z., Cochran, A.J., Gibala, M.J., Gregson, W., Close, G.L., Drust, B., and Morton, J.P. 2013. Reduced carbohydrate availability enhances exerciseinduced p53 signaling in human skeletal muscle: implications for mitochondrial biogenesis. Am. J. Physiol. Regul. Integr. Comp. Physiol. 304(6): R450-8. doi:10.1152/ajpregu.00498.2012.

Benton, C.R., Wright, D.C., and Bonen, A. 2008. PGC-1 $\alpha$-mediated regulation of gene expression and metabolism: implications for nutrition and exercise prescriptions. Appl. Physiol. Nutr. Metab. 33(5): 843-862. doi:10.1139/H08-074.

Bergstrom, J. 1975. Percutaneous needle biopsy of skeletal muscle in physiological and clinical research. Scand. J. Clin. Lab. Invest. 35(7): 609-16.

Bouchard, C., and Rankinen, T. 2001. Individual differences in response to regular physical activity. Med. Sci. Sport. Exerc. 33(6 Suppl): S446-51-NaN-3. doi:10.1097/00005768200106001-00013.

Cartoni, R., Léger, B., Hock, M.B., Praz, M., Crettenand, A., Pich, S., Ziltener, J.-L., Luthi, F., Dériaz, O., Zorzano, A., Gobelet, C., Kralli, A., and Russell, A.P. 2005. Mitofusins 1/2 and ERR $\alpha$ expression are increased in human skeletal muscle after physical exercise. J. Physiol. 567(1): 349-358. doi:10.1113/jphysiol.2005.092031. 
Chomczynski, P., and Sacchi, N. 2006. The single-step method of RNA isolation by acid guanidinium thiocyanate-phenol-chloroform extraction: twenty-something years on. Nat. Protoc. 1(2): 581-585. doi:10.1038/nprot.2006.83.

Crane, J.D., Ogborn, D.I., Cupido, C., Melov, S., Hubbard, A., Bourgeois, J.M., and Tarnopolsky, M.A. 2012. Massage therapy attenuates inflammatory signaling after exerciseinduced muscle damage. Sci. Transl. Med. 4(119): 119ra13. doi:10.1126/scitranslmed.3002882.

Edgett, B.A., Foster, W.S., Hankinson, P.B., Simpson, C.A., Little, J.P., Graham, R.B., and Gurd, B.J. 2013. Dissociation of Increases in PGC-1 $\alpha$ and Its Regulators from Exercise Intensity and Muscle Activation Following Acute Exercise. PLoS One 8(8): e71623. doi:10.1371/journal.pone.0071623.

Egan, B., Carson, B.P., Garcia-Roves, P.M., Chibalin, A. V, Sarsfield, F.M., Barron, N., McCaffrey, N., Moyna, N.M., Zierath, J.R., and O’Gorman, D.J. 2010. Exercise intensitydependent regulation of peroxisome proliferator-activated receptor $\gamma$ coactivator- $1 \alpha$ mRNA abundance is associated with differential activation of upstream signalling kinases in human skeletal muscle. J. Physiol. 588(10): 1779-1790. doi:10.1113/jphysiol.2010.188011.

Field, A. 2013. Discovering Statistics using IBM SPSS Statistics. In 3rd edition. SAGE Publications, London.

Garnier, A., Fortin, D., Zoll, J., N'Guessan, B., Mettauer, B., Lampert, E., Veksler, V., and Ventura-Clapier, R. 2005. Coordinated changes in mitochondrial function and biogenesis in healthy and diseased human skeletal muscle. FASEB J. 19(1): 43-52. doi:10.1096/fj.04- 
$2173 \mathrm{com}$.

Handschin, C., Rhee, J., Lin, J., Tarr, P.T., and Spiegelman, B.M. 2003. An autoregulatory loop controls peroxisome proliferator-activated receptor coactivator 1 expression in muscle. Proc. Natl. Acad. Sci. 100(12): 7111-7116. doi:10.1073/pnas.1232352100.

Jensen, L., Gejl, K.D., Ortenblad, N., Nielsen, J.L., Bech, R.D., Nygaard, T., Sahlin, K., and Frandsen, U. 2015. Carbohydrate restricted recovery from long term endurance exercise does not affect gene responses involved in mitochondrial biogenesis in highly trained athletes. Physiol. Rep. 3(2): e12184-e12184. doi:10.14814/phy2.12184.

Leick, L., Wojtaszewski, J.F.P., Johansen, S.T., Kiilerich, K., Comes, G., Hellsten, Y., Hidalgo, J., and Pilegaard, H. 2007. PGC-1 is not mandatory for exercise- and training-induced adaptive gene responses in mouse skeletal muscle. AJP Endocrinol. Metab. 294(2): E463E474. doi:10.1152/ajpendo.00666.2007.

Little, J.P., Safdar, A., Bishop, D., Tarnopolsky, M.A., and Gibala, M.J. 2011. An acute bout of high-intensity interval training increases the nuclear abundance of PGC-1 and activates mitochondrial biogenesis in human skeletal muscle. AJP Regul. Integr. Comp. Physiol. 300(6): R1303-R1310. doi:10.1152/ajpregu.00538.2010.

Little, J.P., Safdar, A., Cermak, N., Tarnopolsky, M.A., and Gibala, M.J. 2010. Acute endurance exercise increases the nuclear abundance of PGC-1 in trained human skeletal muscle. AJP Regul. Integr. Comp. Physiol. 298(4): R912-R917. doi:10.1152/ajpregu.00409.2009.

Livak, K.J., and Schmittgen, T.D. 2001. Analysis of Relative Gene Expression Data Using RealTime Quantitative PCR and the 2- $\Delta \Delta \mathrm{CT}$ Method. Methods 25(4): 402-408. 
doi:10.1006/meth.2001.1262.

Mootha, V.K., Handschin, C., Arlow, D., Xie, X., St-Pierre, J., Sihag, S., Yang, W., Altshuler, D., Puigserver, P., Patterson, N., Willy, P.J., Schulman, I.G., Heyman, R.A., Lander, E.S., and Spiegelman, B.M. 2004. Erralpha and Gabpa/b specify PGC-1alpha-dependent oxidative phosphorylation gene expression that is altered in diabetic muscle. Proc. Natl. Acad. Sci. U. S. A. 101(17): 6570-6575. doi:10.1073/pnas.0401401101.

Nordsborg, N.B.N.B., Lundby, C.C., Leick, L.L., and Pilegaard, H.H. 2010. Relative workload determines exercise-induced increases in PGC-1alpha mRNA. Med. Sci. Sport. Exerc. 42(8): 1477-1484. doi:10.1249/MSS.0b013e3181d2d21c.

Norrbom, J. 2003. PGC-1 mRNA expression is influenced by metabolic perturbation in exercising human skeletal muscle. J. Appl. Physiol. 96(1): 189-194. doi:10.1152/japplphysiol.00765.2003.

Ogborn, D.I., McKay, B.R., Crane, J.D., Safdar, A., Akhtar, M., Parise, G., and Tarnopolsky, M.A. 2015. Effects of age and unaccustomed resistance exercise on mitochondrial transcript and protein abundance in skeletal muscle of men. AJP Regul. Integr. Comp. Physiol. 308(8): R734-R741. doi:10.1152/ajpregu.00005.2014.

Percival, M.E., Martin, B.J., Gillen, J.B., Skelly, L.E., MacInnis, M.J., Green, A.E., Tarnopolsky, M.A., and Gibala, M.J. 2015. Sodium bicarbonate ingestion augments the increase in PGC-1 $\alpha$ mRNA expression during recovery from intense interval exercise in human skeletal muscle. J. Appl. Physiol. 119(11): 1303-12.

doi:10.1152/japplphysiol.00048.2015. 
Perry, C.G.R., Lally, J., Holloway, G.P., Heigenhauser, G.J.F., Bonen, A., and Spriet, L.L. 2010. Repeated transient mRNA bursts precede increases in transcriptional and mitochondrial proteins during training in human skeletal muscle. J. Physiol. 588(Pt 23): 4795-4810. doi:10.1113/jphysiol.2010.199448.

Philp, A., Hargreaves, M., and Baar, K. 2012. More than a store: regulatory roles for glycogen in skeletal muscle adaptation to exercise. Am. J. Physiol. Endocrinol. Metab. 302(11): E134351. doi:10.1152/ajpendo.00004.2012.

Philp, A., MacKenzie, M.G., Belew, M.Y., Towler, M.C., Corstorphine, A., Papalamprou, A., Hardie, D.G., and Baar, K. 2013. Glycogen Content Regulates Peroxisome Proliferator Activated Receptor- $\partial$ (PPAR- $\partial$ ) Activity in Rat Skeletal Muscle. PLoS One 8(10): 1-8. doi:10.1371/journal.pone.0077200.

Pilegaard, H., Saltin, B., and Neufer, P.D. 2003. Exercise induces transient transcriptional activation of the PGC-1 gene in human skeletal muscle. J. Physiol. 546(3): 851-858. doi:10.1113/jphysiol.2002.034850.

Popov, D. V, Lysenko, E.A., Miller, T.F., Bachinin, A. V, Perfilov, D. V, and Vinogradova, O.L. 2015. The effect of single aerobic exercise on the regulation of mitochondrial biogenesis in skeletal muscles of trained men: A time-course study. Hum. Physiol. 41(3): 296-303. doi:10.1134/S0362119715030123.

Psilander, N., Wang, L., Westergren, J., Tonkonogi, M., and Sahlin, K. 2010. Mitochondrial gene expression in elite cyclists: effects of high-intensity interval exercise. Eur. J. Appl. Physiol. 110(3): 597-606. doi:10.1007/s00421-010-1544-1. 
Puigserver, P., Wu, Z., Park, C.W., Graves, R., Wright, M., and Spiegelman, B.M. 1998. A Cold-Inducible Coactivator of Nuclear Receptors Linked to Adaptive Thermogenesis. Cell 92(6): 829-839. doi:10.1016/S0092-8674(00)81410-5.

Quadrilatero, J., Bombardier, E., Norris, S.M., Talanian, J.L., Palmer, M.S., Logan, H.M., Tupling, A.R., Heigenhauser, G.J.F., and Spriet, L.L. 2010. Prolonged moderate-intensity aerobic exercise does not alter apoptotic signaling and DNA fragmentation in human skeletal muscle. AJP Endocrinol. Metab. 298(3): E534-E547. doi:10.1152/ajpendo.00678.2009.

Ramachandran, B., Yu, G., and Gulick, T. 2008. Nuclear Respiratory Factor 1 Controls Myocyte Enhancer Factor 2A Transcription to Provide a Mechanism for Coordinate Expression of Respiratory Chain Subunits. J. Biol. Chem. 283(18): 11935-11946. doi:10.1074/jbc.M707389200.

Rowe, G.C., El-Khoury, R., Patten, I.S., Rustin, P., and Arany, Z. 2012. PGC-1 $\alpha$ is Dispensable for Exercise-Induced Mitochondrial Biogenesis in Skeletal Muscle. PLoS One 7(7): e41817. doi:10.1371/journal.pone.0041817.

Safdar, A., Little, J.P., Stokl, A.J., Hettinga, B.P., Akhtar, M., and Tarnopolsky, M.A. 2011. Exercise Increases Mitochondrial PGC-1 Content and Promotes Nuclear-Mitochondrial Cross-talk to Coordinate Mitochondrial Biogenesis. J. Biol. Chem. 286(12): 10605-10617. doi:10.1074/jbc.M110.211466.

Saleem, A., and Hood, D.A. 2013. Acute exercise induces tumour suppressor protein p53 translocation to the mitochondria and promotes a p53-Tfam-mitochondrial DNA complex in 
skeletal muscle. J. Physiol. 591(14): 3625-3636. doi:10.1113/jphysiol.2013.252791.

Scarpulla, R.C. 2011. Metabolic control of mitochondrial biogenesis through the PGC-1 family regulatory network. Biochim. Biophys. Acta - Mol. Cell Res. 1813(7): 1269-1278. doi:10.1016/j.bbamcr.2010.09.019.

Serpiello, F.R., Mckenna, M.J., Bishop, D.J., Aughey, R.J., Caldow, M.K., Cameron-Smith, D., and Stepto, N.K. 2012. Repeated sprints alter signaling related to mitochondrial biogenesis in humans. Med. Sci. Sport. Exerc. 44(5): 827-834. doi:10.1249/MSS.0b013e318240067e.

Silvennoinen, M., Ahtiainen, J.P., Hulmi, J.J., Pekkala, S., Taipale, R.S., Nindl, B.C., Laine, T., Häkkinen, K., Selänne, H., Kyröläinen, H., and Kainulainen, H. 2015. PGC-1 isoforms and their target genes are expressed differently in human skeletal muscle following resistance and endurance exercise. Physiol. Rep. 3(10): e12563. doi:10.14814/phy2.12563.

Smith, B.K., Mukai, K., Lally, J.S., Maher, A.C., Gurd, B.J., Heigenhauser, G.J.F., Spriet, L.L., and Holloway, G.P. 2013. AMP-activated protein kinase is required for exercise-induced peroxisome proliferator-activated receptor $\gamma$ co-activator $1 \alpha$ translocation to subsarcolemmal mitochondria in skeletal muscle. J. Physiol. 591(6): 1551-1561. doi:10.1113/jphysiol.2012.245944.

Stepto, N.K., Benziane, B., Wadley, G.D., Chibalin, A. V, Canny, B.J., Eynon, N., and McConell, G.K. 2012. Short-Term Intensified Cycle Training Alters Acute and Chronic Responses of PGC1 $\alpha$ and Cytochrome C Oxidase IV to Exercise in Human Skeletal Muscle. PLoS One 7(12): e53080. doi:10.1371/journal.pone.0053080.

Tachtsis, B., Smiles, W.J., Lane, S.C., Hawley, J.A., and Camera, D.M. 2016. Acute Endurance 
Exercise Induces Nuclear p53 Abundance in Human Skeletal Muscle. Front. Physiol. 7(April). doi:10.3389/fphys.2016.00144.

Virbasius, J. V, and Scarpulla, R.C. 1994. Activation of the human mitochondrial transcription factor A gene by nuclear respiratory factors: a potential regulatory link between nuclear and mitochondrial gene expression in organelle biogenesis. Proc. Natl. Acad. Sci. U. S. A. 91(4): 1309-1313. doi:10.1073/pnas.91.4.1309.

Vollaard, N.B.J., Constantin-Teodosiu, D., Fredriksson, K., Rooyackers, O., Jansson, E., Greenhaff, P.L., Timmons, J.A., and Sundberg, C.J. 2009. Systematic analysis of adaptations in aerobic capacity and submaximal energy metabolism provides a unique insight into determinants of human aerobic performance. J. Appl. Physiol. 106(5): 1479_ 1486. doi:10.1152/japplphysiol.91453.2008.

Wright, D.C., Han, D.-H., Garcia-Roves, P.M., Geiger, P.C., Jones, T.E., and Holloszy, J.O. 2007. Exercise-induced Mitochondrial Biogenesis Begins before the Increase in Muscle PGC-1 Expression. J. Biol. Chem. 282(1): 194-199. doi:10.1074/jbc.M606116200.

Wu, Z., Puigserver, P., Andersson, U., Zhang, C., Adelmant, G., Mootha, V., Troy, A., Cinti, S., Lowell, B., and Scarpulla, R. 1999. Mechanisms Controlling Mitochondrial Biogenesis and Respiration through the Thermogenic Coactivator PGC-1. Cell 98(1): 115-124. doi:10.1016/S0092-8674(00)80611-X. 
Table 1. Participant Characteristics $(N=10)$, mean (SD).

\begin{tabular}{lr}
\hline Age $(\mathrm{yrs})$ & $23(3)$ \\
Weight $(\mathrm{lbs})$ & $160(14)$ \\
$\mathrm{VO}_{2}$ peak $(\mathrm{mL} / \mathrm{kg} / \mathrm{min})$ & $41.8(6.6)$ \\
WR peak $(\mathrm{W})$ & $259(2)$ \\
HR peak (bpm) & $197(1)$ \\
\hline
\end{tabular}

Note: bpm, beats per minute; HR, heart rate; lbs, pounds; min, minute; $\mathrm{mL}$, millilitre; $\mathrm{W}$, watts; yrs, years. 
Table 2. List of Primer Sequences used for Real-Time PCR

\begin{tabular}{lcc}
\hline Gene & Forward Primer (5'-3') & Reverse Primer (5’-3') \\
\hline CS & ACTGTGGACATGATGTATGGTG & GTAGCAGTTTCTGGCATTCAG \\
COXI & TCATAATCGGAGGCTTTGGC & GGTTATGGCAGGGGGTTTTA \\
COXII & CGACTACGGCGGACTAATCT & TCGATTGTCAACGTCAAGGA \\
COXIV & GCAGTGGCGGCAGAATG & AGTCTTCGCTCTTCACAACA \\
PDK4 & CGGCTGGTGGGAAGACTTGA & TGCCGCGGAGTGAAGAGTCT \\
ND1 & AAGTCACCCTAGCCATCATTCTAC & GCAGGAGTAATCAGAGGTGTTCTT \\
ND4 & ACTGGGAGAACTCTCTGTGCTAGT & ATGTAGAGGGAGTATAGGGCTGTG \\
NRF1 & GATGCTTCAGAATTGCCAACC & GTCATCTCACCTCCCTGTAAC \\
PGC-1 $\alpha$ & CACTTACAAGCCAAACCAACAAC & CAATAGTCTTGTTCTCAAATGGGGA \\
PPAR $\alpha$ & CAGGCTATCATTACGGAGTCC & TTCTGTTCTTTTTCTGGATCTTGC \\
TBP & AGACGAGTTCCAGCGCAAGG & GCGTAAGGTGGCAGGCTGTT \\
TFAM & GCAAGTTGTCCAAAGAAACCT & GAAGTTCCCTCCAACGCT \\
\hline
\end{tabular}

Note: COXI from Garnier et al (Garnier et al. 2005); COXII from Little et al (Little et al. 2011); ND1 primer set from Crane et al (Crane et al. 2012), ND4 primer set from Ogborn et al (Ogborn et al. 2015); PGC-1 $\alpha$ primer set from Silvennoien et al (Silvennoinen et al. 2015). 
Table 3. Correlations among changes in mRNA expression of COXIV, COXI, ND1 and ND4.

\begin{tabular}{lcccc}
\hline & COXI & COXII & ND1 & ND4 \\
\hline LO & & & & \\
COXIV & $r=0.89, p=0.00$ & $r=0.69, p=0.03$ & $r=0.72, p=0.02$ & $r=0.73, p=0.02$ \\
COXI & - & $r=0.80, p=0.01$ & $r=0.84, p=0.00$ & $r=0.82, p=0.00$ \\
COXII & - & - & $r=0.91, p=0.00$ & $r=0.91, p=0.00$ \\
ND1 & - & - & - & $r=0.98, p=0.00$ \\
\hline HI & & & & \\
COXIV & $r=0.97, p=0.00$ & $r=0.58, p=0.08$ & $r=0.90, p=0.00$ & $r=0.96, p=0.00$ \\
COXI & - & $r=0.46, p=0.18$ & $r=0.88, p=0.00$ & $r=0.95, p=0.00$ \\
COXII & - & - & $r=0.62, p=0.06$ & $r=0.62, p=0.06$ \\
ND1 & - & - & - & $r=0.95, p=0.00$ \\
\hline
\end{tabular}

Note: COXIV, cytochrome $c$ oxidase subunit IV; COXI, cytochrome $c$ oxidase subunit I; COXII, cytochrome $c$ oxidase subunit II; ND1, NADH dehydrogenase subunit 1; ND4, NADH dehydrogenase subunit 4 . The strength of the relationships are defined as small, medium and large ( $r= \pm 0.10$ to $\pm 0.29 r= \pm 0.30$ to $\pm 0.49 r= \pm 0.50$ to \pm 1.0 , respectively) (Field 2013). 


\section{Figure Captions}

Figure 1. Actual and prescribed kilocalories (A) and the number of intervals performed (B) during the acute bouts of low- (LO) and high-intensity interval exercise (HI). Mean heart rate (C) at the end of each high- (HI; filled circlers) and low- (LO; open circles) intensity interval exercise $(N=10$ except interval $11(n=7)$ and $12(n=1))$. Values presented as mean \pm SD. BPM, beats per minute. $\uparrow$ Significantly $(p<0.05)$ greater than HI, * Significantly $(p<0.05)$ greater than LO at the same interval.

Figure 2. Whole-muscle glycogen content (A) and representative slides of glycogen content (B) before (Pre) and after (Post) an acute bout of low- (LO) and high-intensity interval exercise (HI). Values presented as mean \pm SD. Scale bars represent 100 microns $(\mu \mathrm{m})$. AU, arbitrary units. * Significant $(p<0.05)$ main effect of time. $N=10$.

Figure 3. Effect of an acute bout of low (LO) and high-intensity interval exercise (HI) on the expression of nuclear- (PGC-1 $\alpha$, PPAR $\alpha$, NRF1, TFAM, CS, COXIV) and mitochondrialencoded genes (COXI, COXII, ND1, ND4) at 3-hours post exercise (3hr) (A). The expression of mitochondrial encoded genes (COXI, COXII, ND1, ND4) is coordinated among individual participants in response to $\mathrm{LO}(\mathbf{B})$ and $\mathrm{HI}(\mathbf{C})$. Values presented as mean $\pm \mathrm{SD}$. AU, arbitrary units. * Significantly $(p<0.05)$ greater vs. Pre, $\uparrow$ Significantly $(p<0.05)$ greater vs. LO (paired $t$-tests). $\$$ near significant increase vs. Pre $(p=0.05) . N=10$. 
Figure 1

A

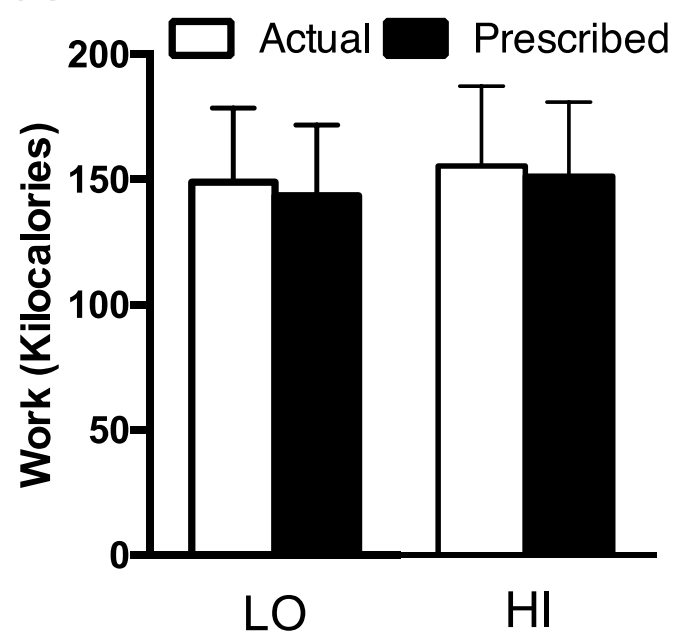

C

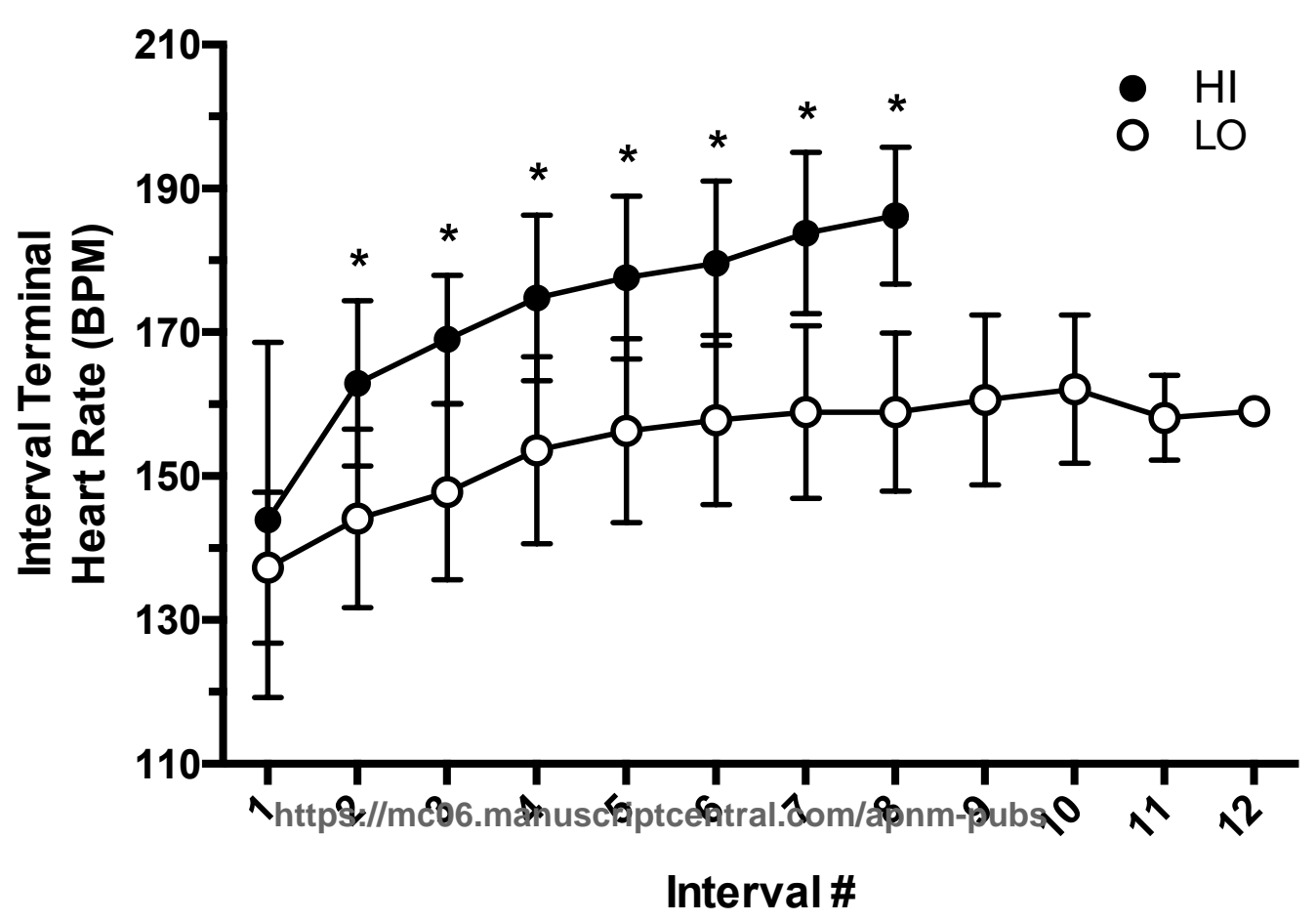

B

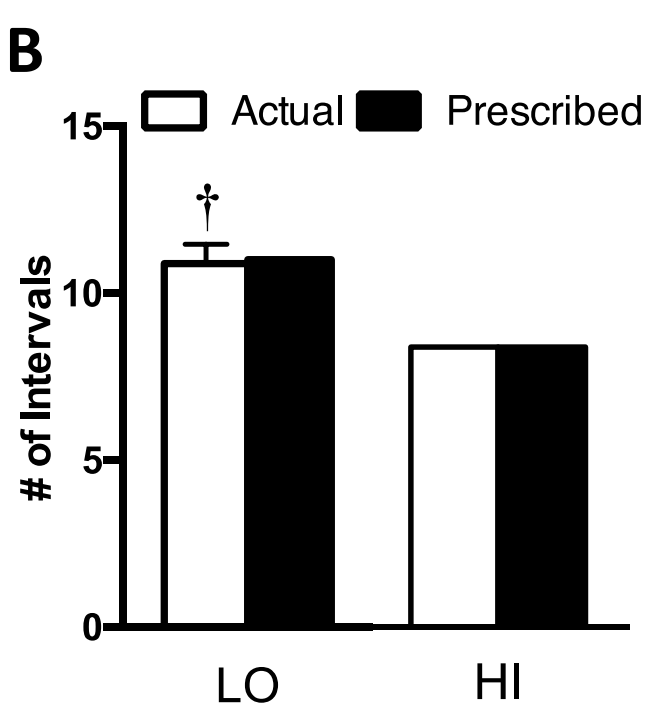

Page 30 of 32 


\section{Figure 2}

A

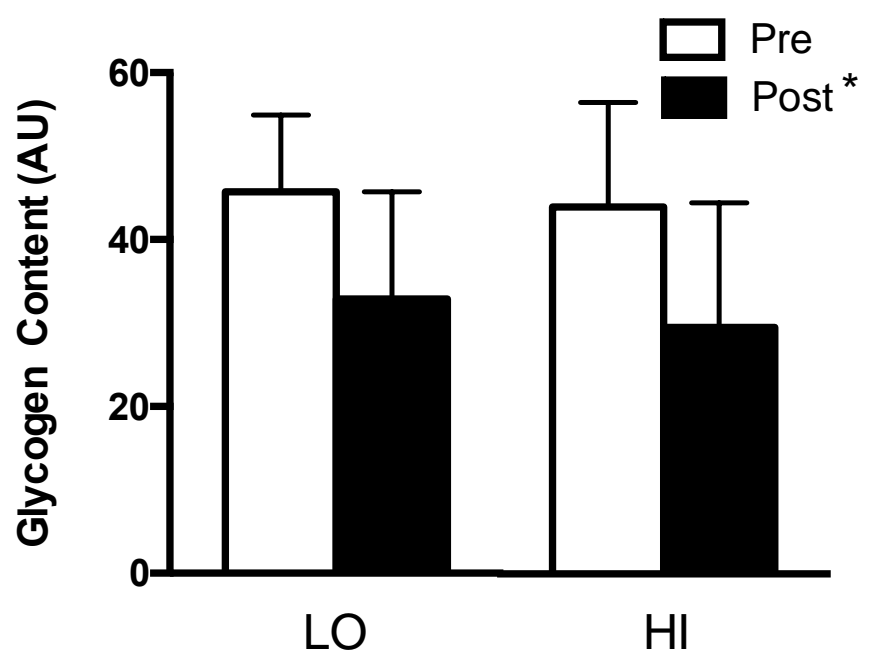

B

Pre

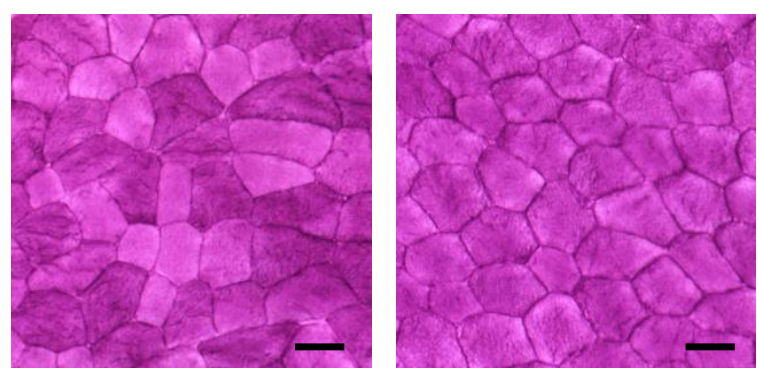

Post 


\section{Figure 3}

A
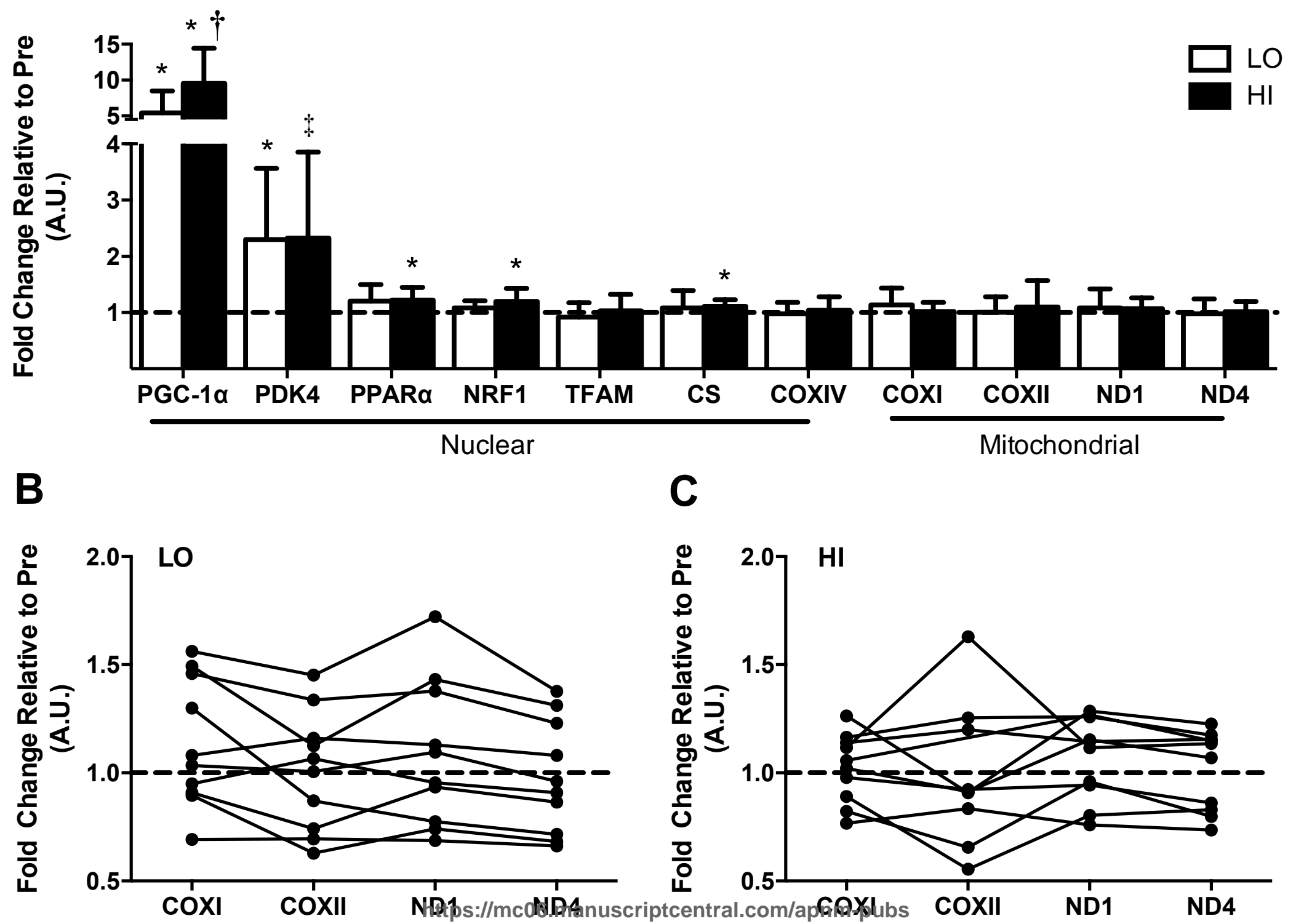